\title{
TRIANGULATED SUBCATEGORIES OF EXTENSIONS, STABLE T-STRUCTURES, AND TRIANGLES OF RECOLLEMENTS
}

\author{
PETER JØRGENSEN AND KIRIKO KATO
}

\begin{abstract}
In a triangulated category $T$ with a pair of triangulated subcategories $X$ and $Y$, one may consider the subcategory of extensions $\mathrm{X} * \mathrm{Y}$.

We give conditions for $\mathrm{X} * \mathrm{Y}$ to be triangulated and use them to provide tools for constructing stable t-structures. In particular, we show how to construct so-called triangles of recollements, that is, triples of stable t-structures of the form $(\mathrm{X}, \mathrm{Y}),(\mathrm{Y}, \mathrm{Z}),(\mathrm{Z}, \mathrm{X})$. We easily recover some triangles of recollements known from the literature.
\end{abstract}

\section{INTRODUCTION}

Let $\mathrm{T}$ be a triangulated category and let $\mathrm{X}, \mathrm{Y} \subseteq \mathrm{T}$ be subcategories. The subcategory of extensions is defined by

$$
\mathrm{X} * \mathrm{Y}=\left\{\begin{array}{l|l}
e \in \mathrm{T} \mid \begin{array}{l}
\text { there is a distinguished triangle } \\
x \rightarrow e \rightarrow y \text { in } \mathrm{T} \text { with } x \in \mathrm{X}, y \in \mathrm{Y}
\end{array}
\end{array}\right\} .
$$

This is a classic object which, as far as we know, was introduced by Beilinson, Bernstein, and Deligne in $[2,1.3 .9]$ and has been used extensively since then, see for instance Bondal-Van den Bergh [3, sec. 2], Iyama-Yoshino [6, sec. 2], and Rouquier [11, sec. 3].

It has been well-known that $\mathrm{X} * \mathrm{Y}$ is triangulated if $\operatorname{Hom}_{\mathrm{T}}(\mathrm{X}, \mathrm{Y})=0$. We have examined this condition to see that a substantial generalization provides exact characterization of $\mathrm{X} * \mathrm{Y}$ to be triangulated.

Theorem A. Let $\mathrm{X}, \mathrm{Y} \subseteq \mathrm{T}$ be triangulated subcategories. The subcategory $\mathrm{X} * \mathrm{Y}$ is triangulated if and only if $\mathrm{Hom}_{\mathrm{T} / \mathrm{X} \cap \mathrm{Y}}(Q \mathrm{X}, Q \mathrm{Y})=0$, where $\mathrm{T} / \mathrm{X} \cap \mathrm{Y}$ is the Verdier quotient and $Q: \mathrm{T} \rightarrow \mathrm{T} / \mathrm{X} \cap \mathrm{Y}$ is the quotient functor.

Note that throughout, all subcategories are full and closed under isomorphisms in the ambient category, and that if $Q: \mathrm{T} \rightarrow \mathrm{T}^{\prime}$ is a functor and $\mathrm{X} \subseteq \mathrm{T}$ a subcategory, then $Q \mathrm{X}$ denotes the isomorphism closure of $\{Q x \mid x \in \mathrm{X}\}$ in $\mathrm{T}^{\prime}$, viewed as a subcategory.

Theorem A is a main ingredient in the proof of Theorem B below which is a tool for constructing stable t-structures. Recall that a stable t-structure in $T$ is a pair $(X, Y)$ of subcategories which are stable under $\Sigma$ and satisfy $\operatorname{Hom}_{\mathrm{T}}(\mathrm{X}, \mathrm{Y})=0$ and $\mathrm{X} * \mathrm{Y}=\mathrm{T}$, see [9, def. 9.14]. Stable t-structures are important in several settings which involve triangulated categories, see for

2010 Mathematics Subject Classification. 18E30, 18E35, 18G35.

Key words and phrases. Homotopy category, quotient category, recollement, stable t-structure, subcategory of extensions, triangle of recollements, triangulated category. 
instance [5]. In particular, they play a prominent role in algebraic geometry where they are known as semi-orthogonal decompositions, see for instance [4].

Note that if $\mathrm{X}, \ldots, \mathrm{Y} \subseteq \mathrm{T}$ are subcategories, then $\langle\mathrm{X}, \ldots, \mathrm{Y}\rangle$ denotes the smallest triangulated subcategory containing them.

Theorem B. Each row in the following table defines a triangulated subcategory $\mathrm{U} \subseteq \mathrm{T}$ and considers the quotient functor $Q: \mathrm{T} \rightarrow \mathrm{T} / \mathrm{U}$. It then shows one or more stable t-structures in $\mathrm{T} / \mathrm{U}$.

\begin{tabular}{c|c|c|c}
$\begin{array}{c}\text { Given triangulated } \\
\text { subcategories of } \mathrm{T} \ldots\end{array}$ & Which satisfy... & Define... & $\begin{array}{c}\text { Then there are stable } \\
\text { t-structures in } \mathrm{T} / \mathrm{U} \ldots\end{array}$ \\
\hline $\mathrm{X}, \mathrm{Y}$ & $\mathrm{X} * \mathrm{Y}=\mathrm{T}$ & $\mathrm{U}=\mathrm{X} \cap \mathrm{Y}$ & $(Q \mathrm{X}, Q \mathrm{Y})$ \\
\hline $\mathrm{X}, \mathrm{Y}, \mathrm{Z}$ & $\mathrm{X} * \mathrm{Y}=\mathrm{T}$ & $\mathrm{U}=\langle\mathrm{X} \cap \mathrm{Y}, \mathrm{Y} \cap \mathrm{Z}\rangle$ & $(Q \mathrm{X}, Q \mathrm{Y})$ \\
& $\mathrm{Y} * \mathrm{Z}=\mathrm{T}$ & & $(Q \mathrm{Y}, Q \mathrm{Z})$ \\
\hline $\mathrm{X}, \mathrm{Y}, \mathrm{Z}$ & $\mathrm{X} * \mathrm{Y}=\mathrm{T}$ & & $(Q \mathrm{X}, Q \mathrm{Y})$ \\
& $\mathrm{Y} * \mathrm{Z}=\mathrm{T}$ & $\mathrm{U}=\langle\mathrm{X} \cap \mathrm{Y}, \mathrm{Y} \cap \mathrm{Z}, \mathrm{Z} \cap \mathrm{X}\rangle$ & $(Q \mathrm{Y}, Q \mathrm{Z})$ \\
$(Q \mathrm{Z}, Q \mathrm{X})$
\end{tabular}

Note that the penultimate row of the table gives two "adjacent" stable t-structures, and that this is equivalent to giving a recollement, see [5, prop. 1.8]. The last row gives three "cyclically adjacent" stable t-structures. In the terminology of [5, def. 1.9], the triple ( $Q \mathrm{X}, Q \mathrm{Y}, Q \mathrm{Z})$ is a "triangle of recollements". It is somewhat surprising that such a structure is possible, but it provides a setup with a large number of pleasant symmetries, see for instance [5, props. 1.10 and 1.16]. We show in Section 6 how Theorem B easily recovers two triangles of recollements known from [5, thm. 5.8] and [8, thm. 2.10].

Our third main theorem is a variation on a part of Theorem B; it is also proved by means of Theorem A. Note that a thick subcategory is a triangulated subcategory closed under direct summands. If $(\mathrm{X}, \mathrm{Y})$ is a stable t-structure, then $\mathrm{X}$ and $\mathrm{Y}$ are thick by [9, prop. 9.15].

Theorem C. Let $\mathrm{U} \subseteq \mathrm{T}$ be a thick subcategory, $Q: \mathrm{T} \rightarrow \mathrm{T} / \mathrm{U}$ the quotient functor. There is a bijection

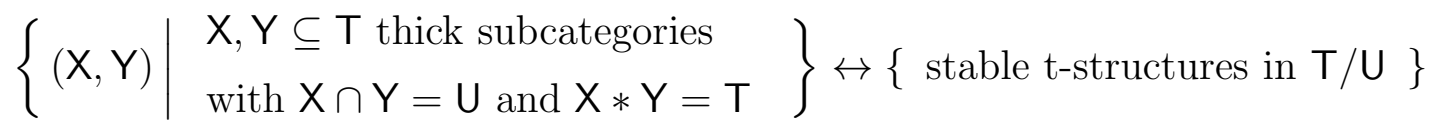

given by $(\mathrm{X}, \mathrm{Y}) \mapsto(Q \mathrm{X}, Q \mathrm{Y})$.

The paper is organised as follows: Section 1 gives the proof of Theorem A. Section 2 proves some lemmas needed later. Sections 3 and 4 give the proofs of Theorems B and C. Section 
5 gives another result based on Theorem A, and Section 6 uses Theorem B to recover two triangles of recollements known from the literature.

\section{Proof of Theorem A}

We shall begin with the following observation which helps us to prove Theorem A.

Lemma 1.1. Let $\mathrm{X}, \mathrm{Y} \subseteq \mathrm{T}$ be triangulated subcategories. The following conditions are equivalent.

(i) $\mathrm{X} * \mathrm{Y}$ is triangulated.

(ii) $\mathrm{Y} * \mathrm{X} \subseteq \mathrm{X} * \mathrm{Y}$.

(iii) Each morphism $x \rightarrow y$ with $x \in \mathrm{X}, y \in \mathrm{Y}$ factors through an object from $\mathrm{X} \cap \mathrm{Y}$.

(i) $\Leftrightarrow$ (ii): (i) $\Rightarrow$ (ii) is obvious. (ii) $\Rightarrow(\mathrm{i})$ : Since $\mathrm{Y} * \mathrm{X} \subseteq \mathrm{X} * \mathrm{Y}$, we can compute as follows using that $*$ is associative by $[2$, lem. 1.3.10].

$$
(\mathrm{X} * \mathrm{Y}) *(\mathrm{X} * \mathrm{Y})=\mathrm{X} *(\mathrm{Y} * \mathrm{X}) * \mathrm{Y} \subseteq \mathrm{X} *(\mathrm{X} * \mathrm{Y}) * \mathrm{Y}=(\mathrm{X} * \mathrm{X}) *(\mathrm{Y} * \mathrm{Y})=\mathrm{X} * \mathrm{Y}
$$

So $\mathrm{X} * \mathrm{Y}$ is closed under extensions, and since it is also closed under $\Sigma^{ \pm 1}$, it is triangulated.

We should mention (i) $\Leftrightarrow$ (ii) immediately follows also from Lemma 2.1 since (i) equivalently says $X * Y=\langle X, Y\rangle$.

(ii) $\Rightarrow$ (iii): Let $x \rightarrow y$ be a morphism with $x \in \mathrm{X}, y \in \mathrm{Y}$. Completing to a distinguished triangle $x \rightarrow y \rightarrow c$, we have $c \in \mathrm{Y} * \mathrm{X} \subseteq \mathrm{X} * \mathrm{Y}$, so there is a distinguished triangle $x_{1} \rightarrow c \rightarrow y_{1}$ with $x_{1} \in \mathrm{X}, y_{1} \in \mathrm{Y}$. The octahedral axiom provides a commutative diagram where rows and columns are distinguished triangles.

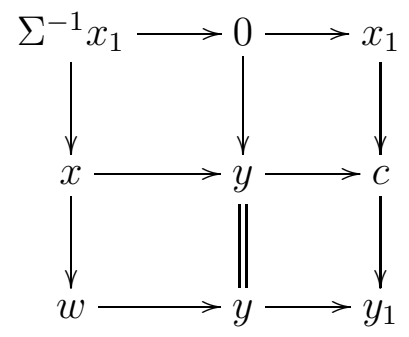

The first column shows $w \in \mathbf{X}$ and the last row shows $w \in \mathrm{Y}$, so $x \rightarrow w \rightarrow y$ is a factorization as claimed in (iii).

(iii) $\Rightarrow$ (ii): If $e \in \mathrm{Y} * \mathrm{X}$ is given then there is a distinguished triangle $y \rightarrow e \rightarrow x \stackrel{\xi}{\rightarrow} \Sigma y$ with $y \in \mathrm{Y}, x \in \mathrm{X}$. Use (iii) to factorise $\xi$ as $x \rightarrow w \rightarrow \Sigma y$ with $w \in \mathrm{X} \cap \mathrm{Y}$. The octahedral axiom 
again gives a commutative diagram where rows and columns are distinguished triangles.

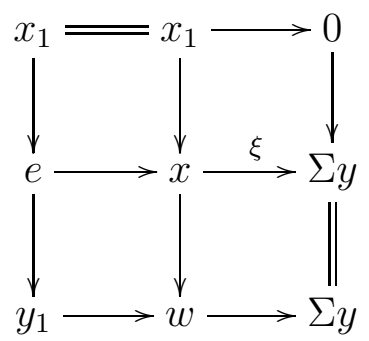

The second column shows $x_{1} \in \mathrm{X}$ and the last row shows $y_{1} \in \mathrm{Y}$, so the first column shows $e \in \mathrm{X} * \mathrm{Y}$.

Remark 1.2. The equivalence between (i) and (ii) is a triangulated analogue of [13, thm. 3.2] on Serre subcategories of module categories.

Proof of Theorem A. It suffices to show that (iii) of Lemma 1.1 is equivalent to the following.

(iv) We have $\operatorname{Hom}_{\mathrm{T} / \mathrm{X} \cap \mathrm{Y}}(Q \mathrm{X}, Q \mathrm{Y})=0$, where $\mathrm{T} / \mathrm{X} \cap \mathrm{Y}$ is the Verdier quotient and $Q: \mathrm{T} \rightarrow$ $\mathrm{T} / \mathrm{X} \cap \mathrm{Y}$ is the quotient functor.

Indeed, this is due to Verdier. Namely, using his terminology, condition (iii) is equivalent to $\mathrm{Y}$ being "X-localisante à droite" by [12, prop. II.2.3.5(a)]. By [12, prop. II.2.3.5(c)] this is equivalent to $\mathrm{Y} / \mathrm{X} \cap \mathrm{Y}$ consisting of objects which are " $R$-libre à droite" where $R: \mathrm{T} / \mathrm{X} \cap \mathrm{Y} \rightarrow \mathrm{T} / \mathrm{X}$ is the quotient functor. Finally, this is equivalent to condition (iv) by [12, prop. II.2.3.3(a)].

Remark 1.3. We could have added the following conditions to Lemma 1.1

(v) If $\mathrm{X}^{\prime} \subseteq \mathrm{T}$ is a triangulated subcategory such that $\mathrm{X} \cap \mathrm{Y} \subseteq \mathrm{X}^{\prime} \subseteq \mathrm{X}$, then $\mathrm{X}^{\prime} * \mathrm{Y}$ is triangulated.

(vi) If $\mathrm{Y}^{\prime} \subseteq \mathrm{T}$ is a triangulated subcategory such that $\mathrm{X} \cap \mathrm{Y} \subseteq \mathrm{Y}^{\prime} \subseteq \mathrm{Y}$, then $\mathrm{X} * \mathrm{Y}^{\prime}$ is triangulated.

Namely, there are implications as follows between these and conditions (i)-(iii) of Lemma 1.1 and (iv) in the proof of Theorem A.

(iii) $\Rightarrow(\mathrm{v})$ : It is enough to see $\mathrm{Y} * \mathrm{X}^{\prime} \subseteq \mathrm{X}^{\prime} * \mathrm{Y}$, but this follows by a small variation of the proof of (iii) $\Rightarrow$ (ii) in Lemma 1.1 .

(iii) $\Rightarrow(\mathrm{vi})$ is similar, and $(\mathrm{v}) \Rightarrow(\mathrm{i})$ and $(\mathrm{vi}) \Rightarrow(\mathrm{i})$ are trivial.

The following consequence of Theorem A is well-known, but we do not know a published reference.

Corollary 1.4. If $\mathrm{X}, \mathrm{Y} \subseteq \mathrm{T}$ are triangulated subcategories with $\operatorname{Hom}_{\mathrm{T}}(\mathrm{X}, \mathrm{Y})=0$, then $\mathrm{X} * \mathrm{Y}$ is triangulated. 
Proof. Since $\operatorname{Hom}_{\mathrm{T}}(\mathrm{X}, \mathrm{Y})=0$ implies that $\mathrm{X} \cap \mathrm{Y}$ is trivial, $Q: \mathrm{T} \rightarrow \mathrm{T} / \mathrm{X} \cap \mathrm{Y}$ is an equivalence. Hence $\operatorname{Hom}_{\mathrm{T} / \mathrm{X} \cap \mathrm{Y}}(Q \mathrm{X}, Q \mathrm{Y})=\operatorname{Hom}_{\mathrm{T}}(\mathrm{X}, \mathrm{Y})=0$. Due to Theorem $\mathrm{A}, \mathrm{X} * \mathrm{Y}$ is triangulated.

\section{LEMMAS}

The following lemma uses the notation $\mathrm{X}^{* n}=\mathrm{X} * \cdots * \mathrm{X}$ with $\mathrm{X}$ repeated $n$ times.

Lemma 2.1. If $\mathrm{X}, \mathrm{Y} \subseteq \mathrm{T}$ are subcategories closed under $\Sigma^{ \pm 1}$ and containing 0 , then

$$
\langle\mathrm{X}, \mathrm{Y}\rangle=\bigcup_{n \geq 1}(\mathrm{X} * \mathrm{Y})^{* n}
$$

Proof. The inclusion $\supseteq$ is clear and $\subseteq$ holds because the right hand side contains $\mathrm{X}$ and $\mathrm{Y}$, and is closed under $\Sigma^{ \pm 1}$ and extensions so is triangulated.

Lemma 2.2. Let $\mathrm{X}^{\prime} \subseteq \mathrm{X} \subseteq \mathrm{T}$ and $\mathrm{Y}^{\prime} \subseteq \mathrm{Y} \subseteq \mathrm{T}$ be triangulated subcategories such that $\mathrm{X} * \mathrm{Y}$ is triangulated. Then

(i) $X \cap\left\langle X^{\prime}, Y\right\rangle=\left\langle X^{\prime}, X \cap Y\right\rangle$,

(ii) $\left\langle X, Y^{\prime}\right\rangle \cap Y=\left\langle X \cap Y, Y^{\prime}\right\rangle$.

Proof. Part (ii) is clear from part (i) by symmetry. In (i) the inclusion $\supseteq$ is clear.

To prove $\subseteq$ in (i), it is enough by Lemma 2.1 to show $\mathrm{X} \cap\left(\mathrm{Y} * \mathrm{X}^{\prime}\right)^{* n} \subseteq\left\langle\mathrm{X}^{\prime}, \mathrm{X} \cap \mathrm{Y}\right\rangle$ for each $n \geq 1$. We do so by showing the stronger statement

$$
Q_{n}= \begin{cases}\text { (a) } \mathrm{X} \cap\left(\mathrm{Y} * \mathrm{X}^{\prime}\right)^{* n} \subseteq\left\langle\mathrm{X}^{\prime}, \mathrm{X} \cap \mathrm{Y}\right\rangle, \\ \text { (b) } \mathrm{X} \cap\left(\left(\mathrm{Y} * \mathrm{X}^{\prime}\right)^{* n} * \mathrm{Y}\right) \subseteq\left\langle\mathrm{X}^{\prime}, \mathrm{X} \cap \mathrm{Y}\right\rangle\end{cases}
$$

for each $n \geq 1$ by induction.

$n=1$ : To show $Q_{1}$, part (a), let $e \in \mathrm{X} \cap\left(\mathrm{Y} * \mathrm{X}^{\prime}\right)$ be given. There is a distinguished triangle $y \rightarrow e \rightarrow x^{\prime}$ with $y \in \mathbf{Y}, x^{\prime} \in \mathbf{X}^{\prime}$. Since $e$ and $x^{\prime}$ are both in $\mathbf{X}$, so is $y$, so we have $y \in \mathbf{X} \cap \mathbf{Y}$ whence the distinguished triangle shows $e \in(\mathrm{X} \cap \mathrm{Y}) * \mathrm{X}^{\prime} \subseteq\left\langle\mathrm{X}^{\prime}, \mathrm{X} \cap \mathrm{Y}\right\rangle$.

To show $Q_{1}$, part $(\mathrm{b})$, let $e \in \mathrm{X} \cap\left(\left(\mathrm{Y} * \mathrm{X}^{\prime}\right) * \mathrm{Y}\right)$ be given. Since $*$ is associative we have $e \in \mathrm{Y} *\left(\mathbf{X}^{\prime} * \mathbf{Y}\right)$ so there is a distinguished triangle $y \rightarrow e \rightarrow a$ with $y \in \mathbf{Y}, a \in \mathbf{X}^{\prime} * \mathbf{Y}$. There is also a distinguished triangle $x_{1}^{\prime} \rightarrow a \rightarrow y_{1}$ with $x_{1}^{\prime} \in \mathrm{X}^{\prime}, y_{1} \in \mathrm{Y}$.

We have $e \in \mathbf{X}, y_{1} \in \mathbf{Y}$, so by Lemma 1.1 the composition $e \rightarrow a \rightarrow y_{1}$ factors as $e \rightarrow w \rightarrow y_{1}$ for an object $w \in \mathrm{X} \cap \mathrm{Y}$. The 9-lemma [9, prop. 4.9] now provides a commutative diagram where rows and columns are distinguished triangles.

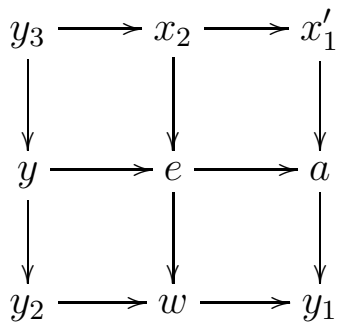


The second column shows $x_{2} \in \mathrm{X}$. The third row shows $y_{2} \in \mathrm{Y}$, whence the first column shows $y_{3} \in \mathrm{Y}$ and the first row shows $x_{2} \in \mathrm{Y} * \mathbf{X}^{\prime}$. Hence $x_{2} \in \mathbf{X} \cap\left(\mathrm{Y} * \mathbf{X}^{\prime}\right)$ whence $Q_{1}$, part (a) shows $x_{2} \in\left\langle\mathbf{X}^{\prime}, \mathrm{X} \cap \mathrm{Y}\right\rangle$. Then the second column shows $e \in\left\langle\mathrm{X}^{\prime}, \mathrm{X} \cap \mathrm{Y}\right\rangle$ as desired.

$n \geq 2$ : To show $Q_{n}$, part (a), let $e \in \mathrm{X} \cap\left(\mathrm{Y} * \mathrm{X}^{\prime}\right)^{* n}$ be given. Since $*$ is associative there is a distinguished triangle $y \rightarrow e \rightarrow a$ with $y \in \mathrm{Y}, a \in\left(\mathrm{X}^{\prime} * \mathrm{Y}\right)^{*(n-1)} * \mathrm{X}^{\prime}$. There is also a distinguished triangle $b \rightarrow a \rightarrow x^{\prime}$ with $b \in\left(\mathbf{X}^{\prime} * \mathrm{Y}\right)^{*(n-1)}, x^{\prime} \in \mathbf{X}^{\prime}$. The octahedral axiom provides a commutative diagram where rows and columns are distinguished triangles.

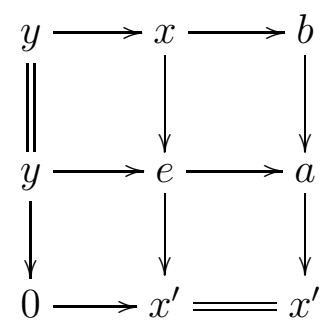

The second column shows $x \in \mathrm{X}$ and the first row shows $x \in \mathrm{Y} *\left(\mathrm{X}^{\prime} * \mathrm{Y}\right)^{*(n-1)}=\left(\mathrm{Y} * \mathrm{X}^{\prime}\right)^{*(n-1)} * \mathrm{Y}$. Statement $Q_{n-1}$, part (b), hence shows $x \in\left\langle\mathrm{X}^{\prime}, \mathrm{X} \cap \mathrm{Y}\right\rangle$, and then the second column shows $e \in\left\langle\mathrm{X}^{\prime}, \mathrm{X} \cap \mathrm{Y}\right\rangle$ as desired.

To show $Q_{n}$, part $(\mathrm{b})$, let $e \in \mathrm{X} \cap\left(\left(\mathrm{Y} * \mathrm{X}^{\prime}\right)^{* n} * \mathrm{Y}\right)$ be given. Since $*$ is associative there is a distinguished triangle $y \rightarrow e \rightarrow a$ with $y \in \mathrm{Y}, a \in\left(\mathrm{X}^{\prime} * \mathrm{Y}\right)^{* n}$. There is also a distinguished triangle $b \rightarrow a \rightarrow y_{1}$ with $b \in \mathrm{X}^{\prime} *\left(\mathrm{Y} * \mathrm{X}^{\prime}\right)^{*(n-1)}, y_{1} \in \mathrm{Y}$.

As above, the composition $e \rightarrow a \rightarrow y_{1}$ factors as $e \rightarrow w \rightarrow y_{1}$ for an object $w \in \mathbf{X} \cap \mathrm{Y}$, and we obtain another commutative diagram where rows and columns are distinguished triangles.

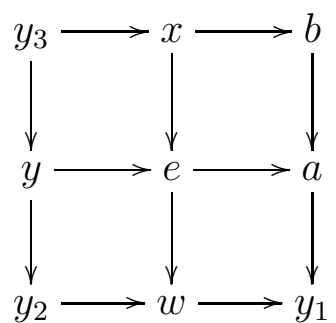

The second column shows $x \in \mathrm{X}$. The third row shows $y_{2} \in \mathrm{Y}$ whence the first column shows $y_{3} \in \mathrm{Y}$ and the first row shows $x \in\left(\mathrm{Y} * \mathrm{X}^{\prime}\right)^{* n}$. Hence $x \in \mathrm{X} \cap\left(\mathrm{Y} * \mathrm{X}^{\prime}\right)^{* n}$ whence $Q_{n}$, part (a) shows $x \in\left\langle\mathrm{X}^{\prime}, \mathrm{X} \cap \mathrm{Y}\right\rangle$. The second column shows $e \in\left\langle\mathrm{X}^{\prime}, \mathrm{X} \cap \mathrm{Y}\right\rangle$ as desired.

If $Q: \mathrm{T} \rightarrow \mathrm{T}^{\prime}$ is a functor and $\mathrm{X}^{\prime} \subseteq \mathrm{T}^{\prime}$ is a subcategory then $Q^{-1} \mathrm{X}^{\prime}=\left\{t \in \mathrm{T} \mid Q t \in \mathrm{X}^{\prime}\right\}$ will be viewed as a subcategory of $\mathrm{X}$.

Remark 2.3. If $\mathrm{U} \subseteq \mathrm{T}$ is a thick subcategory, then $\mathrm{X} \mapsto Q \mathrm{X}$ gives a bijection

$$
\left\{\begin{array}{l}
\text { triangulated subcategories } \mathrm{X} \subseteq \mathrm{T} \\
\text { with } \mathrm{U} \subseteq \mathrm{X} \subseteq \mathrm{T}
\end{array}\right\} \leftrightarrow\{\text { triangulated subcategories of } \mathrm{T} / \mathrm{U}\}
$$

with inverse $\mathrm{Y} \mapsto Q^{-1} \mathrm{Y}$. Under the bijection, thick subcategories correspond to thick subcategories. See [12, prop. II.2.3.1].

Lemma 2.4. Let $\mathrm{U} \subseteq \mathrm{T}$ be a thick subcategory and let $Q: \mathrm{T} \rightarrow \mathrm{T} / \mathrm{U}$ be the quotient functor. 
(i) If $\mathrm{X}, \mathrm{Y} \subseteq \mathrm{T}$ are triangulated subcategories with $\mathrm{U} \subseteq \mathrm{X} \cap \mathrm{Y}$, then

(a) $Q(\mathrm{X} \cap \mathrm{Y})=Q \mathrm{X} \cap Q \mathrm{Y}$,

(b) $Q(\mathrm{X} * \mathrm{Y})=Q \mathrm{X} * Q \mathrm{Y}$.

(ii) If $\mathrm{X} \subseteq \mathrm{T}$ is any subcategory then $Q^{-1} Q \mathrm{X}=\mathrm{U} * \mathrm{X} * \mathrm{U}$.

Proof. (i.a): The inclusion $\subseteq$ is clear. To see $\supseteq$, let $Q z \in Q \mathrm{X} \cap Q \mathrm{Y}$ be given. Then $Q z \in Q \mathrm{X}$ so $z \in Q^{-1} Q \mathrm{X}=\mathrm{X}$ where we used $\mathrm{U} \subseteq \mathrm{X}$ and Remark 2.3. Similarly we get $z \in \mathrm{Y}$ so $z \in \mathrm{X} \cap \mathrm{Y}$ whence $Q z \in Q(\mathrm{X} \cap \mathrm{Y})$.

(i.b): The inclusion $\subseteq$ is clear. To see $\supseteq$, let $Q z \in Q \mathrm{X} * Q \mathrm{Y}$ be given. There is a distinguished triangle $Q x \rightarrow Q z \rightarrow Q y$ in $\mathrm{T} / \mathrm{U}$ with $x \in \mathrm{X}, y \in \mathrm{Y}$. By [9, def. 8.4] the triangle has the form $Q(a \rightarrow c \rightarrow b)$ for a distinguished triangle $a \rightarrow c \rightarrow b$ in T. Then $Q a \cong Q x$ so $Q a \in Q \mathrm{X}$, and as in the proof of (i.a) we get $a \in \mathbf{X}$. Similarly we get $b \in \mathrm{Y}$ so $c \in \mathbf{X} * \mathrm{Y}$ whence $Q z \cong Q c \in Q(\mathrm{X} * \mathrm{Y})$.

(ii): The inclusion $\supseteq$ follows from $Q(\mathrm{U} * \mathrm{X} * \mathrm{U})=Q(\mathrm{U}) * Q(\mathrm{X}) * Q(\mathrm{U})$ and $Q \mathrm{U}=0$; indeed, $Q$ is an exact functor and $\mathrm{U}$ is the kernel of $Q$ by [12, cor. II.2.2.11(a)].

To see $\subseteq$, let $z \in Q^{-1} Q \mathrm{X}$ be given. This means $Q z \in Q \mathrm{X}$ so there is an isomorphism $Q x \stackrel{\sim}{\rightarrow} Q z$ with $x \in \mathrm{X}$. By [10, def. 2.1.11], the isomorphism is represented by a diagram $x \stackrel{\psi}{\leftarrow} w \stackrel{\chi}{\rightarrow} z$ in $\mathrm{T}$ with cone $(\psi) \in \mathrm{U}$. That is, the isomorphism equals $(Q \chi)(Q \psi)^{-1}$. Since $Q \psi$ and $(Q \chi)(Q \psi)^{-1}$ are isomorphisms, so is $Q \chi$, and it follows that $0 \cong \operatorname{cone}(Q \chi) \cong Q$ (cone $\chi)$ whence $\operatorname{cone}(\chi) \in \mathrm{U}$. From $x \in \mathbf{X}$ and cone $(\psi) \in \mathbf{U}$ follows $w \in \mathbf{U} * \mathbf{X}$, and together with $\operatorname{cone}(\chi) \in \mathbf{U}$ this shows $z \in \mathrm{U} * \mathrm{X} * \mathrm{U}$.

\section{Proof of Theorem B}

Before the proof, recall again that a stable t-structure in $\mathrm{T}$ is a pair $(\mathrm{X}, \mathrm{Y})$ of subcategories which are stable under $\Sigma$ and satisfy $\operatorname{Hom}_{\mathrm{T}}(\mathrm{X}, \mathrm{Y})=0$ and $\mathrm{X} * \mathrm{Y}=\mathrm{T}$.

Proof of Theorem B, the first row of the table, in which we have triangulated subcategories $\mathrm{X}, \mathrm{Y} \subseteq \mathrm{T}$ satisfying $\mathrm{X} * \mathrm{Y}=\mathrm{T}$. We set $\mathrm{U}=\mathrm{X} \cap \mathrm{Y}$ and consider the quotient functor $Q: \mathrm{T} \rightarrow \mathrm{T} / \mathrm{U}$.

We must show that $(Q \mathrm{X}, Q \mathrm{Y})$ is a stable t-structure in $\mathrm{T} / \mathrm{U}$.

Since $\mathrm{X}, \mathrm{Y}$ are closed under $\Sigma$ in $\mathrm{T}$, it follows that $Q \mathrm{X}, Q \mathrm{Y}$ are closed under $\Sigma$ in $\mathrm{T} / \mathrm{U}$. Moreover, $\mathrm{X} * \mathrm{Y}=\mathrm{T}$ clearly implies $Q \mathrm{X} * Q \mathrm{Y}=\mathrm{T} / \mathrm{U}$. Finally, Theorem A implies Hom $\mathrm{H}_{\mathrm{U}}(Q \mathrm{X}, Q \mathrm{Y})=0$.

Proof of Theorem B, the second row of the table, in which we have triangulated subcategories $\mathrm{X}, \mathrm{Y}, \mathrm{Z} \subseteq \mathrm{T}$ satisfying $\mathrm{X} * \mathrm{Y}=\mathrm{Y} * \mathrm{Z}=\mathrm{T}$. We set $\mathrm{U}=\langle\mathrm{X} \cap \mathrm{Y}, \mathrm{Y} \cap \mathrm{Z}\rangle$ and consider the quotient functor $Q: \mathrm{T} \rightarrow \mathrm{T} / \mathrm{U}$.

We must show that the pairs $(Q \mathrm{X}, Q \mathrm{Y})$ and $(Q \mathrm{Y}, Q \mathrm{Z})$ are stable t-structures in $\mathrm{T} / \mathrm{U}$. It suffices to show a proof for the first pair as the second is similar.

Set

$$
\mathrm{Y}^{\prime}=\mathrm{Y} \cap \mathrm{Z}, \quad \widetilde{\mathrm{X}}=\left\langle\mathrm{X}, \mathrm{Y}^{\prime}\right\rangle .
$$

Since $\mathrm{Y}^{\prime} \subseteq \mathrm{U}$ we have $Q \widetilde{\mathrm{X}}=Q \mathrm{X}$, so it is enough to show that $(Q \widetilde{\mathrm{X}}, Q \mathrm{Y})$ is a stable t-structure. 
Since $\widetilde{X}, Y$ are closed under $\Sigma$ in $\mathrm{T}$, it follows that $Q \widetilde{\mathrm{X}}, Q \mathrm{Y}$ are closed under $\Sigma$ in $\mathrm{T} / \mathrm{U}$. Moreover, $\mathrm{X} * \mathrm{Y}=\mathrm{T}$ implies $\widetilde{\mathrm{X}} * \mathrm{Y}=\mathrm{T}$ whence $Q \widetilde{\mathrm{X}} * Q \mathrm{Y}=\mathrm{T} / \mathrm{U}$ is clear. Finally, we have $\mathrm{Y}^{\prime} \subseteq \mathrm{Y}$ whence Lemma 2.2(ii) gives the second "=" in

$$
\widetilde{X} \cap \mathrm{Y}=\left\langle\mathrm{X}, \mathrm{Y}^{\prime}\right\rangle \cap \mathrm{Y}=\left\langle\mathrm{X} \cap \mathrm{Y}, \mathrm{Y}^{\prime}\right\rangle=\langle\mathrm{X} \cap \mathrm{Y}, \mathrm{Y} \cap \mathrm{Z}\rangle=\mathrm{U}
$$

Since $\widetilde{\mathrm{X}} * \mathrm{Y}=\mathrm{T}$ is triangulated, Theorem A implies $\operatorname{Hom}_{\mathrm{T} / \mathrm{U}}(Q \widetilde{\mathrm{X}}, Q \mathrm{Y})=0$.

Proof of Theorem B, the third row of the table, in which we have triangulated subcategories $\mathrm{X}, \mathrm{Y}, \mathrm{Z} \subseteq \mathrm{T}$ satisfying $\mathrm{X} * \mathrm{Y}=\mathrm{Y} * \mathrm{Z}=\mathrm{Z} * \mathrm{X}=\mathrm{T}$. We set $\mathrm{U}=\langle\mathrm{X} \cap \mathrm{Y}, \mathrm{Y} \cap \mathrm{Z}, \mathrm{Z} \cap \mathrm{X}\rangle$ and consider the quotient functor $Q: \mathrm{T} \rightarrow \mathrm{T} / \mathrm{U}$.

We must show that the pairs $(Q \mathrm{X}, Q \mathrm{Y}),(Q \mathrm{Y}, Q \mathrm{Z}),(Q \mathrm{Z}, Q \mathrm{X})$ are stable t-structures in T/U. It suffices to show a proof for the first pair as the others are similar.

Indeed, this can be shown analogously to the previous case by setting

$$
\begin{array}{ll}
Y^{\prime}=Y \cap Z, & \widetilde{X}=\left\langle X, Y^{\prime}\right\rangle \\
X^{\prime}=Z \cap X, & \widetilde{Y}=\left\langle Y, X^{\prime}\right\rangle .
\end{array}
$$

Then $Q \widetilde{\mathrm{X}}=Q \mathrm{X}, Q \widetilde{\mathrm{Y}}=Q \mathrm{Y}$, and it is enough to show that $(Q \widetilde{\mathrm{X}}, Q \widetilde{\mathrm{Y}})$ is a stable t-structure.

As above, $Q \widetilde{\mathrm{X}}, Q \widetilde{\mathrm{Y}}$ are closed under $\Sigma$ in $\mathrm{T} / \mathrm{U}$ and $Q \widetilde{\mathrm{X}} * Q \widetilde{\mathrm{Y}}=\mathrm{T} / \mathrm{U}$. Finally, since $\mathrm{X}^{\prime} \subseteq \mathrm{X}, \mathrm{Y}^{\prime} \subseteq \mathrm{Y}$, a computation based on Lemma 2.2 (i and ii) gives $\widetilde{X} \cap \widetilde{Y}=U$. As above, Theorem A then implies $\operatorname{Hom}_{\mathrm{T} / \mathrm{U}}(Q \widetilde{\mathrm{X}}, Q \widetilde{\mathrm{Y}})=0$.

\section{Proof of Theorem C}

When $\mathrm{X}, \mathrm{Y} \subseteq \mathrm{T}$ are thick subcategories satisfying $\mathrm{X} \cap \mathrm{Y}=\mathrm{U}$ and $\mathrm{X} * \mathrm{Y}=\mathrm{T}$, the first item of the table in Theorem B shows that $(Q \mathrm{X}, Q \mathrm{Y})$ is a stable t-structure in $\mathrm{T} / \mathrm{U}$. Hence $(\mathrm{X}, \mathrm{Y}) \mapsto$ $(Q \mathrm{X}, Q \mathrm{Y})$ is indeed a map

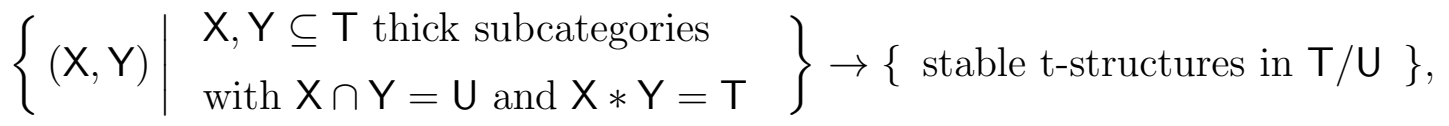

as claimed in Theorem C.

The map (4.1) is injective because of Remark 2.3.

The map (4.1) is surjective: A stable t-structure in $\mathrm{T} / \mathrm{U}$ consists of two thick subcategories. By Remark 2.3 it has the form $(Q \mathrm{X}, Q \mathrm{Y})$ for two unique thick subcategories $\mathrm{X}, \mathrm{Y}$ satisfying $\mathrm{U} \subseteq \mathrm{X} \cap \mathrm{Y} \subseteq \mathrm{T}$. To complete the proof, we must show $\mathrm{X} \cap \mathrm{Y}=\mathrm{U}$ and $\mathrm{X} * \mathrm{Y}=\mathrm{T}$.

Lemma 2.4(i.a) gives $Q(\mathrm{X} \cap \mathrm{Y})=Q \mathrm{X} \cap Q \mathrm{Y}=0$. This implies $\mathrm{X} \cap \mathrm{Y}=\mathrm{U}$ by Remark 2.3.

Note that as a consequence,

$$
\operatorname{Hom}_{\mathrm{T} / \mathrm{X} \cap \mathrm{Y}}(Q \mathrm{X}, Q \mathrm{Y})=\operatorname{Hom}_{\mathrm{T} / \mathrm{U}}(Q \mathrm{X}, Q \mathrm{Y})=0
$$

so $\mathrm{X} * \mathrm{Y}$ is triangulated by Theorem $\mathrm{A}$.

Lemma 2.4(i.b) gives $Q(\mathrm{X} * \mathrm{Y})=Q \mathrm{X} * Q \mathrm{Y}=\mathrm{T} / \mathrm{U}=Q \mathrm{~T}$. This implies $\mathrm{X} * \mathrm{Y}=\mathrm{T}$ by Remark 2.3 since $\mathrm{X} * \mathrm{Y}$ is a triangulated subcategory satisfying $\mathrm{U} \subseteq \mathrm{X} * \mathrm{Y} \subseteq \mathrm{T}$. 


\section{Another Result on subCATEGories of EXtensions}

Theorem 5.1. Let $\mathrm{X}, \mathrm{Y}, \mathrm{V}$ be thick subcategories of $\mathrm{T}$ satisfying $\mathrm{X} \cap \mathrm{Y} \subseteq \mathrm{V} \subseteq \mathrm{T}$ and $\mathrm{X} * \mathrm{Y}=\mathrm{T}$. The following are equivalent.

(i) $\mathrm{X} * \mathrm{~V}$ is triangulated.

(ii) $\mathrm{V} * \mathrm{Y}$ is triangulated.

(iii) $\mathrm{V}=(\mathrm{X} \cap \mathrm{V}) *(\mathrm{Y} \cap \mathrm{V})$.

Proof. Write $\mathrm{U}=\mathrm{X} \cap \mathrm{Y}$ and let $Q: \mathrm{T} \rightarrow \mathrm{T} / \mathrm{U}$ be the quotient functor. Note that $Q \mathrm{X}, Q \mathrm{Y}, Q \mathrm{~V}$ are thick (and in particular triangulated) subcategories of $\mathrm{T} / \mathrm{U}$ by Remark 2.3.

We claim that each condition in the theorem is equivalent to the corresponding condition in the following list.

(i') $Q \mathrm{X} * Q \mathrm{~V}$ is triangulated.

(ii') $Q \mathrm{~V} * Q \mathrm{Y}$ is triangulated.

(iii') $Q \mathrm{~V}=(Q \mathrm{X} \cap Q \mathrm{~V}) *(Q \mathrm{Y} \cap Q \mathrm{~V})$.

(i) $\Leftrightarrow\left(i^{\prime}\right)$ : By Lemma 1.1 this amounts to

$$
\mathrm{V} * \mathrm{X} \subseteq \mathrm{X} * \mathrm{~V} \Leftrightarrow Q \mathrm{~V} * Q \mathrm{X} \subseteq Q \mathrm{X} * Q \mathrm{~V}
$$

But $\Rightarrow$ follows from Lemma 2.4(i.b) and $\Leftarrow$ can be seen as follows using Lemma 2.4(i.b and ii).

$$
\begin{aligned}
Q \mathrm{~V} * Q \mathrm{X} \subseteq Q \mathrm{X} * Q \mathrm{~V} & \Leftrightarrow Q(\mathrm{~V} * \mathrm{X}) \subseteq Q(\mathrm{X} * \mathrm{~V}) \\
& \Rightarrow Q^{-1} Q(\mathrm{~V} * \mathrm{X}) \subseteq Q^{-1} Q(\mathrm{X} * \mathrm{~V}) \\
& \Rightarrow \mathrm{U} * \mathrm{~V} * \mathrm{X} * \mathrm{U} \subseteq \mathrm{U} * \mathrm{X} * \mathrm{~V} * \mathrm{U} \\
& \Leftrightarrow \mathrm{V} * \mathrm{X} \subseteq \mathrm{X} * \mathrm{~V} .
\end{aligned}
$$

The last $\Leftrightarrow$ holds since $\mathrm{U} \subseteq \mathrm{V}$ and $\mathrm{U} \subseteq \mathrm{X}$.

(ii) $\Leftrightarrow($ ii') and (iii) $\Leftrightarrow($ iii'): Similar.

We now show that (i') through (iii') are equivalent.

$\left(\mathrm{i}^{\prime}\right) \Rightarrow($ iii'): The inclusion $\supseteq$ is obvious. We shall show $\subseteq$. The condition $\mathrm{X} * \mathrm{Y}=\mathrm{T}$ clearly implies $Q \mathrm{X} * Q \mathrm{Y}=\mathrm{T} / \mathrm{U}$ so given $Q v \in Q \mathrm{~V}$ there is a distinguished triangle

$$
Q x \rightarrow Q v \rightarrow Q y \rightarrow \Sigma Q x
$$

in $\mathrm{T} / \mathrm{U}$ with $Q x \in Q \mathrm{X}, Q y \in Q \mathrm{Y}$. This shows $Q y \in Q \mathrm{~V} * Q \mathrm{X}$ but (i') means $Q \mathrm{~V} * Q \mathrm{X} \subseteq Q \mathrm{X} * Q \mathrm{~V}$ by Lemma 1.1, so $Q y \in Q \mathrm{X} * Q \mathrm{~V}$. Hence there is a distinguished triangle $Q x_{1} \rightarrow Q y \rightarrow Q v_{1}$ with $Q x_{1} \in Q \mathrm{X}, Q v_{1} \in Q \mathrm{~V}$, but $\mathrm{Hom}_{\mathrm{T} / \mathrm{U}}(Q \mathrm{X}, Q \mathrm{Y})=0$ by Theorem A so the triangle splits whence $Q y$ is a direct summand of $Q v_{1}$. Since $Q \vee$ is thick this implies $Q y \in Q \vee$. The distinguished triangle (5.1) then shows $Q x \in Q \vee$. We have shown $Q x \in Q \mathrm{X} \cap Q \vee, Q y \in Q \mathrm{Y} \cap Q \vee$ so (5.1) shows (iii').

(iii' $) \Rightarrow\left(\right.$ i'$\left.^{\prime}\right)$ : When (iii') holds we have

$Q \mathrm{X} * Q \mathrm{~V}=Q \mathrm{X} *((Q \mathrm{X} \cap Q \mathrm{~V}) *(Q \mathrm{Y} \cap Q \mathrm{~V}))=(Q \mathrm{X} *(Q \mathrm{X} \cap Q \mathrm{~V})) *(Q \mathrm{Y} \cap Q \mathrm{~V})=Q \mathrm{X} *(Q \mathrm{Y} \cap Q \mathrm{~V})$ 
so it is enough to see that $Q \mathrm{X} *(Q \mathrm{Y} \cap Q \mathrm{~V})$ is triangulated. However, $Q \mathrm{X}$ and $Q \mathrm{Y} \cap Q \mathrm{~V}$ are both triangulated by Remark 2.3 and $\operatorname{Hom}_{\mathrm{T} / \mathrm{U}}(Q \mathrm{X}, Q \mathrm{Y} \cap Q \mathrm{~V})=0$ because $\operatorname{Hom}_{\mathrm{T} / \mathrm{U}}(Q \mathrm{X}, Q \mathrm{Y})=0$ by Theorem A. Hence $Q \mathrm{X} *(Q \mathrm{Y} \cap Q \mathrm{~V})$ is triangulated by Corollary 1.4.

$($ ii') $\Leftrightarrow($ iii') follows by similar arguments.

\section{EXAMPLES}

6.a. The homotopy category of projective modules. Let $R$ be an Iwanaga-Gorenstein ring, that is, a noetherian ring which has finite injective dimension from either side as a module over itself. Let $\mathrm{T}=\mathrm{K}_{(\mathrm{b})}(\operatorname{Prj} R)$ be the homotopy category of complexes of projective right- $R$ modules with bounded homology. Define subcategories of $\mathrm{T}$ by

$$
\mathrm{X}=\mathrm{K}_{(\mathrm{b})}^{-}(\operatorname{Prj} R), \quad \mathrm{Y}=\mathrm{K}_{\mathrm{ac}}(\operatorname{Prj} R), \quad \mathrm{Z}=\mathrm{K}_{(\mathrm{b})}^{+}(\operatorname{Prj} R)
$$

where $\mathrm{K}_{(\mathrm{b})}^{-}(\operatorname{Prj} R)$ is the isomorphism closure of the class of complexes $P$ with $P^{i}=0$ for $i \gg 0$ and $\mathrm{K}_{(\mathrm{b})}^{+}(\operatorname{Prj} R)$ is defined analogously with $i \ll 0$, while $\mathrm{K}_{\mathrm{ac}}(\operatorname{Prj} R)$ is the subcategory of acyclic (that is, exact) complexes.

Note that $\mathrm{Y}$ is equal to $\mathrm{K}_{\mathrm{tac}}(\operatorname{Prj} R)$, the subcategory of totally acyclic complexes, that is, acyclic complexes which stay acyclic under the functor $\operatorname{Hom}_{R}(-, Q)$ when $Q$ is projective, see [7, cor. 5.5 and par. 5.12].

By [5, prop. 2.3(1), lem. 5.6(1), and rmk. 5.14] there are stable t-structures $(\mathrm{X}, \mathrm{Y}),(\mathrm{Y}, \mathrm{Z})$ in $\mathrm{T}$, SO

$$
\mathrm{X} * \mathrm{Y}=\mathrm{Y} * \mathrm{Z}=\mathrm{T}, \mathrm{X} \cap \mathrm{Y}=\mathrm{Y} \cap \mathrm{Z}=0 .
$$

If $P \in \mathrm{T}$ is given, then there is a distinguished triangle $P^{\geq 0} \rightarrow P \rightarrow P^{<0}$ where $P^{\geq 0}$ and $P^{<0}$ are the hard truncations. Since $P^{\geq 0} \in \mathbf{Z}$ and $P^{<0} \in \mathbf{X}$, we have

$$
\mathrm{Z} * \mathrm{X}=\mathrm{T} \text {. }
$$

Finally,

$$
\mathrm{Z} \cap \mathrm{X}=\mathrm{K}_{(\mathrm{b})}^{+}(\operatorname{Prj} R) \cap \mathrm{K}_{(\mathrm{b})}^{-}(\operatorname{Prj} R)=\mathrm{K}^{\mathrm{b}}(\operatorname{Prj} R)
$$

is the isomorphism closure of the class of bounded complexes. If we use an obvious shorthand for quotient categories, then the last row of the table in Theorem B now provides a triangle of recollements

$$
\left(\mathrm{K}_{(\mathrm{b})}^{-} / \mathrm{K}^{\mathrm{b}}(\operatorname{Prj} R), \mathrm{K}_{\mathrm{ac}}(\operatorname{Prj} R), \mathrm{K}_{(\mathrm{b})}^{+} / \mathrm{K}^{\mathrm{b}}(\operatorname{Prj} R)\right)
$$

in $\mathrm{K}_{(\mathrm{b})} / \mathrm{K}^{\mathrm{b}}(\operatorname{Prj} R)$. The reason that we can write $\mathrm{K}_{\mathrm{ac}}(\operatorname{Prj} R)$ instead of its image in $\mathrm{K}_{(\mathrm{b})} / \mathrm{K}^{\mathrm{b}}(\operatorname{Prj} R)$ is that the two are equivalent by [5, prop. 1.5].

This example and its analogue with finitely generated projective modules were first obtained in [5, thms. 2.8 and 5.8] and motivated the definition of triangles of recollements. 
6.b. The symmetric Auslander category. Let $R$ be a noetherian commutative ring with a dualizing complex. In [8, def. 2.1] we introduced the symmetric Auslander category $\mathrm{A}^{\mathrm{s}}(R)$ as the isomorphism closure in $\mathrm{K}_{(\mathrm{b})}(\operatorname{Prj} R)$ of the class of complexes $P$ for which there exist $T, U \in \mathrm{K}_{\text {tac }}(\operatorname{Prj} R)$ such that $P^{\ll 0}=T^{\ll 0}$ and $P^{\gg 0}=U^{\gg 0}$.

The methods of $[8$, sec. 2] show that there are stable t-structures

$$
\left(\mathrm{A}(R), \mathrm{K}_{\mathrm{tac}}(\operatorname{Prj} R)\right),\left(\mathrm{K}_{\mathrm{tac}}(\operatorname{Prj} R), S(\mathrm{~B}(R))\right)
$$

in $\mathrm{A}^{\mathrm{s}}(R)$ with $S(\mathrm{~B}(R)) * \mathrm{~A}(R)=\mathrm{A}^{\mathrm{s}}(R)$. Here $\mathrm{A}(R)$ and $\mathrm{B}(R)$ are the Auslander and Bass categories of $R$, see [1, sec. 3], and $S$ is the functor introduced in [7, sec. 4.3]. Since $\mathrm{A}(R)$ (resp. $S(\mathrm{~B}(R))$ ) consists of right-bounded (resp. left-bounded) complexes $P$ such that $P^{\ll 0}=T^{\ll 0}$ (resp. $P^{\gg 0}=T^{\gg 0}$ ) for some $T \in \mathrm{K}_{\text {tac }}(\operatorname{Prj} R)$ [8, Rem. 2.2], we have $\mathrm{A}(R) \cap S(\mathrm{~B}(R))=$ $\mathrm{K}^{\mathrm{b}}(\operatorname{Prj} R)$.

With $\mathrm{T}=\mathrm{A}^{\mathrm{s}}(R)$ and

$$
\mathrm{X}=\mathrm{A}(R), \quad \mathrm{Y}=\mathrm{K}_{\text {tac }}(\operatorname{Prj} R), \quad \mathrm{Z}=S(\mathrm{~B}(R)),
$$

the last row of the table in Theorem $\mathrm{B}$ recovers the triangle of recollements

$$
\left(\mathrm{A}(R) / \mathrm{K}^{\mathrm{b}}(\operatorname{Prj} R), \mathrm{K}_{\mathrm{tac}}(\operatorname{Prj} R), S(\mathrm{~B}(R)) / \mathrm{K}^{\mathrm{b}}(\operatorname{Prj} R)\right)
$$

in $\mathrm{A}^{\mathrm{s}}(R) / \mathrm{K}^{\mathrm{b}}(\operatorname{Prj} R)$ first obtained in $[8$, thm. 2.10]. As in the previous example, the category $\mathrm{K}_{\text {tac }}(\operatorname{Prj} R)$ is equivalent to its image in $\mathrm{A}^{\mathrm{s}}(R) / \mathrm{K}^{\mathrm{b}}(\operatorname{Prj} R)$ so we can write $\mathrm{K}_{\text {tac }}(\operatorname{Prj} R)$ instead of the image.

Acknowledgement. We gratefully acknowledge support from JSPS Grant 22540053 and a Scheme 2 Grant from the London Mathematical Society.

\section{REFERENCES}

[1] L. L. Avramov and H.-B. Foxby, Ring homomorphisms and finite Gorenstein dimension, Proc. London Math. Soc. (3) 75 (1997), 241-270.

[2] A. A. Beilinson, J. Bernstein, and P. Deligne, "Faisceaux pervers", Astérisque 100 (1982) (Vol. 1 of the proceedings of the conference "Analysis and topology on singular spaces", Luminy, 1981).

[3] A. Bondal and M. Van den Bergh, Generators and representability of functors in commutative and noncommutative geometry, Mosc. Math. J. 3 (2003), 1-36.

[4] D. Huybrechts, "Fourier-Mukai transforms in algebraic geometry", Oxford Mathematical Monographs, Oxford University Press, Oxford, 2006.

[5] O. Iyama, K. Kato, and J. Miyachi, Recollement of homotopy categories and Cohen-Macaulay modules, J. K-Theory 8 (2011), 507-542.

[6] O. Iyama and Y. Yoshino, Mutation in triangulated categories and rigid Cohen-Macaulay modules, Invent. Math. 172 (2008), 117-168.

[7] S. Iyengar and H. Krause, Acyclicity versus total acyclicity for complexes over noetherian rings, Doc. Math. 11 (2006), 207-240.

[8] P. Jørgensen and K. Kato, Symmetric Auslander and Bass categories, Math. Proc. Cambridge Phil. Soc. 250 (2011), 227-240.

[9] J. Miyachi, Derived Categories with applications to representations of algebras, Chiba University, 2000. Available at the author's webpage: http://www.u-gakugei.ac.jp/ miyachi/seminar.html.

[10] A. Neeman, "Triangulated categories", Ann. of Math. Stud., Vol. 148, Princeton University Press, Princeton, 2001.

[11] R. Rouquier, Dimensions of triangulated categories, J. K-Theory 1 (2008), 193-256. 
[12] J.-L. Verdier, "Des catégories dérivées des catégories abéliennes", with a preface by Luc Illusie; edited and with a note by Georges Maltsiniotis, Astérisque 239 (1996).

[13] T. Yoshizawa, Subcategories of extension modules by Serre subcategories, Proc. Amer. Math. Soc. 140 (2012), 2293-2305.

School of Mathematics and Statistics, Newcastle University, Newcastle upon Tyne Ne1 7RU, UNITED KingDOM

E-mail address: peter.jorgensen@ncl.ac.uk

$U R L:$ http://www.staff.ncl.ac.uk/peter.jorgensen

Department of Mathematics and Information Sciences, Osaka Prefecture University, Osaka, JAPAN

E-mail address: kiriko@mi.s.osakafu-u.ac.jp

$U R L:$ http://www.mi.s.osakafu-u.ac.jp/ kiriko 